\title{
Analysis of Nutritional Status and Levels of Sebum on Various Age Groups
}

\author{
Farida Tabri $^{1}$, Ilhamjaya Patellongi ${ }^{2}$, Siswanto Wahab ${ }^{1}$, Khairuddin Djawad ${ }^{1}$ \\ ${ }^{1}$ Department of Dermatology and Venereology, Faculty of Medicine, Hasanuddin University, Makassar, Indonesia \\ ${ }^{2}$ Department of Physiology, Faculty of Public Health, Hasanuddin University, Makassar, Indonesia
}

\section{Email address:}

farida_tabri@yahoo.com (F. Tabri)

\section{To cite this article:}

Farida Tabri, Ilhamjaya Patellongi, Siswanto Wahab, Khairuddin Djawad. Analysis of Nutritional Status and Levels of Sebum on Various Age Groups. American Journal of Clinical and Experimental Medicine. Vol. 5, No. 1, 2017, pp. 26-29. doi: 10.11648/j.ajcem.20170501.16

Received: January 18, 2017; Accepted: February 3, 2017; Published: February 27, 2017

\begin{abstract}
The effects of aging on skin physiology have been reported previously. In this study we tried to elucidate the correlation of skin types with age and nutritional status. There is a correlation between the age groups with nutritional status, correlation between age groups with the sebum levels on the forehead, and also there is a correlation between nutritional status and sebum level on forehead.
\end{abstract}

Keywords: Nutritional Status, Aging, Sebum Level, Skin Type

\section{Introduction}

Diet and lifestyle, coupled with maintenance of a healthy body weight are important in the maintenance of health for all age groups but are crucial for healthy aging. Maintaining a good nutritional status has significant implications for health and wellbeing, delaying and reducing the risk of developing disease, maintaining functional independence and thus promoting continued independent living [1].

Aging is accompanied by many changes that can make it more difficult for nutritional needs to be met. These changes have been categorised into broad categories of physical/physiological and psychosocial [1].

Aging is accompanied by an increased likelihood of suffering from one, or more, chronic diseases such as respiratory disease, arthritis, stroke, depression and dementia. These conditions may affect appetite, functional ability or ability to swallow, all leading to altered food intake and impairment of nutritional status [1].

Medications used in the treatment of chronic illness can also have a detrimental effect on nutritional status through loss of appetite, nausea, diarrhoea, reduced gastrointestinal motility and dry mouth [1].

Taste and smell diminish with age and poor dentition may limit food choice to soft foods. Dry mouth (xerostomia) is common, making swallowing difficult with subsequent avoidance of foods. Malabsorption of essential nutrient may result as a result of gastrointestinal changes such as atrophic gastritis. Gastric emptying slows with aging with a potential detrimental effect on appetite. All of these factors, independently or collectively, can lead to a reduction in food intake [1].

Today, food insecurity is of the major public health issues and food security is considered to be the health index of household and individual. Food insecurity and hunger can bring about adverse social and mental effects as well as undesirable physical ones [2].

As we age body composition changes - fat mass increases and lean body mass (muscle) decreases (sarcopenia). Loss of muscle mass begins at around age 50 but becomes more accelerated after the age of 60 years of age, and fat mass continues to increase until around the age of 75 years. Loss of muscle mass leads to a reduction in basal metabolic rate by approximately $15 \%$ between the age of 30 and 80 , and this results in a subsequent reduction in energy requirements, of around $150 \mathrm{kcal}$ per day after the age of 75 [1].

While many older adults remain healthy and eat well, those in poorer health may experience difficulties in meeting their nutritional needs. Meeting the diet and nutrition needs of older people is crucial for the maintenance of health, functional independence and quality of life [1].

The skin protects the body from mechanical damage, microbial invasion, and radiation or UV light, as a barrier 
between the internal part of the body and the external environment. Thus, the skin plays an important role in the maintenance of homeostasis of the body by minimizing the moisture loss and regulating the temperature [3].

Sebaceous glands are found over most of the skin surface, although there are few on the hands and feet and none on the palms and soles. Sebaceous glands on the mid-back, forehead and chin are larger and more numerous than elsewhere (up to 400-900 glands per square centimeter). They are also numerous in the ear canal and around the genitals [4].

The most obvious function of the sebaceous gland is to excrete sebum. Sebum is a mixture of relatively non-polar lipids, most of which are synthesized de novo by the sebaceous glands. The composition of sebum is remarkably species and age specific. Human sebaceous glands secrete a lipid mixture containing squalene and wax esters, as well as cholesterol esters, triglycerides and possibly some free cholesterol [5].

Most sebaceous glands open out into the hair follicle. Sebum is produced by sebaceous glands when they disintegrate. Sebum is primarily composed of triglycerides $(\sim 41 \%)$, wax esters $(\sim 26 \%)$, squalene $(\sim 12 \%)$, and free fatty acids $(\sim 16 \%)$. Sebum has the following functions; it reduces water loss from the skin surface, protects the skin from infection by bacteria and fungi and colonized by the bacteria Proprionibacterium acnes, which may have a role in immune system regulation $[6,7]$.

Sebum secretions vary individually according to age, sex, inherited traits, and topographical variations of the skin. In general, facial skin types are classified into three types according to an individual's subjective judgment, as: oily, normal, or dry facial skin types. However, an individual's feelings on the topic of facial sebum secretion are too subjective, and significant discrepancies exist between such subjective descriptions of facial skin type and objective measurements [8].

In this study we tried to elucidate the correlation of skin types with age and nutritional status. Because problems related to skin health and skin condition can originate from the internal factors, it is necessary to study nutritional factor that affects skin condition, the nutritional status for instance. Thus, this study was conducted to utilize the study results as basic data in the skin care and health area.

\section{Subjects and Methods}

\subsection{Subjects}

Subjects were a convenient sample recruited in a nursing home for women, church community, and students in Hasanuddin University area. 100 Indonesian female volunteers were included in this study. The inclusion criteria used were that subjects were healthy and cooperative as the study was conducted through a medical history questionnaire. Questionnaires were administered in order to collect information about age, nutritional status, the usage of air conditioner, recent dermatological symptoms, and allergic history. Evaluation of the skin types was classified according to the sebum level measured by Sebumeter. Sebumeter is a well acceptable photometric method in measuring sebum levels by the "European Expert Group on Efficacy Measurement of Cosmetics and Other Topical Products (EEMCO) guidance for the in vivo assessment of skin greasiness" [9].

Sebumeter (SM815, Courage \& Khazaka, Köln or Cologne, Germany). Two different sites of the skin surface were selected that is the forehead (mid-glabella) and the back of the hand (dorsal). Participants were asked not to use any cosmetics and not to wash within $2 \mathrm{~h}$ of measurements. Sebum was collected from each site on a plastic strip using a constant pressure of $10 \mathrm{~N}$ for $30 \mathrm{~s}$. The Sebumeter SM 815 was calibrated to zero with an unused tape section prior to each measurement. Sebum secretion amounts were recorded and mean sebum excretions were calculated. All procedures were performed by the same investigator in a room at constant temperature of $23^{\circ} \mathrm{C}$ and measurements were conducted in December 2016. The patient skin types were determined using the sebum secretion guidelines supplied by the Sebumeter.

This study examined the correlation of skin types with age, nutritional status, disease history, the usage of air conditioner, and allergic history by using Sebumeter and classification suggested by Youn et al $[8,10]$. The reference values used to evaluate the facial skin types are listed in Table 1.

Table 1. Reference values for the evaluation of facial skin type by sebum secretion measured with Sebumeter.

\begin{tabular}{ll}
\hline Skin type & Mean Sebum Excretions* $\left(\boldsymbol{\mu g} / \mathbf{c m}^{2}\right)$ \\
\hline Dry & $<88$ \\
Normal & $88-204$ \\
Oily & $>204$ \\
\hline
\end{tabular}

*The reference values were calculated using reference values of sebum secretion suggested by manufacturer

\subsection{BMI}

The height and weight of the subjects were measured manually and formulated into BMI in order to assess the nutritional status of the subjects.

The measurement of body weight with various corrections for height is frequently used to assess risk for obesity-related diseases because it is the most economical and practical approach in both clinical and epidemiologic settings [11]. The National Institutes of Health (NIH) and the World Health Organization (WHO) have adopted similar body weight guidelines for overweight and obesity. The most commonly used measure is Quetelet's index or BMI, defined as squared height divided by weight, with weight in kilograms and height in meters. Values of body weight adjusted for height, referred to as body mass index (BMI; in $\left.\mathrm{kg} / \mathrm{m}^{2}\right)$. The BMI thresholds are for underweight $(<18.5)$, overweight $(\geq 25)$, and obesity $(\geq 30)$. However, BMI does not take into account the relative proportions of fat and lean tissue and cannot distinguish the location of fat distribution $[12,13]$. 


\subsection{Methods}

The base of this study was using observation methods with cross sectional study approach, and all data resulted in this study were statistically analyzed by using Statistic
Application and were presented as mean and standard deviation by skin type. The relationships among sebum levels on the forehead, nutritional status and age groups were examined through correlation analysis.

\section{Results}

Table 2. Sebumeter Results on 100 skin samples at various age groups, nutritional state, air conditioner usage and allergic history.

\begin{tabular}{|c|c|c|c|c|c|c|c|}
\hline \multirow{3}{*}{ Age group } & \multirow{2}{*}{\multicolumn{4}{|c|}{ Nutritional State (BMI) }} & \multicolumn{3}{|l|}{ Sebumeter Level } \\
\hline & & & & & \multirow{2}{*}{$\begin{array}{l}\text { Oily } \\
\text { Fore Head }(n=1)\end{array}$} & \multirow{2}{*}{$\begin{array}{l}\text { Normal } \\
\begin{array}{l}\text { Fore Head } \\
(n=19)\end{array} \\
\end{array}$} & \multirow{2}{*}{$\begin{array}{l}\text { Dry } \\
\begin{array}{l}\text { Fore Head } \\
(n=80)\end{array} \\
\end{array}$} \\
\hline & Obese $(n=7)$ & Over Weight $(n=28)$ & Healthy $(n=52)$ & Under Weight $(n=13)$ & & & \\
\hline $21-30(n=20)$ & 2 & 4 & 11 & 3 & 1 & 4 & 15 \\
\hline $31-40(n=20)$ & 1 & 6 & 12 & 1 & 0 & 5 & 15 \\
\hline $41-50(n=20)$ & 3 & 10 & 7 & 0 & 0 & 7 & 13 \\
\hline $51-60(n=20)$ & 1 & 7 & 12 & 0 & 0 & 1 & 19 \\
\hline TOTAL $(n=100)$ & 7 & 28 & 52 & 13 & 1 & 19 & 80 \\
\hline
\end{tabular}

Table 2. Continue.

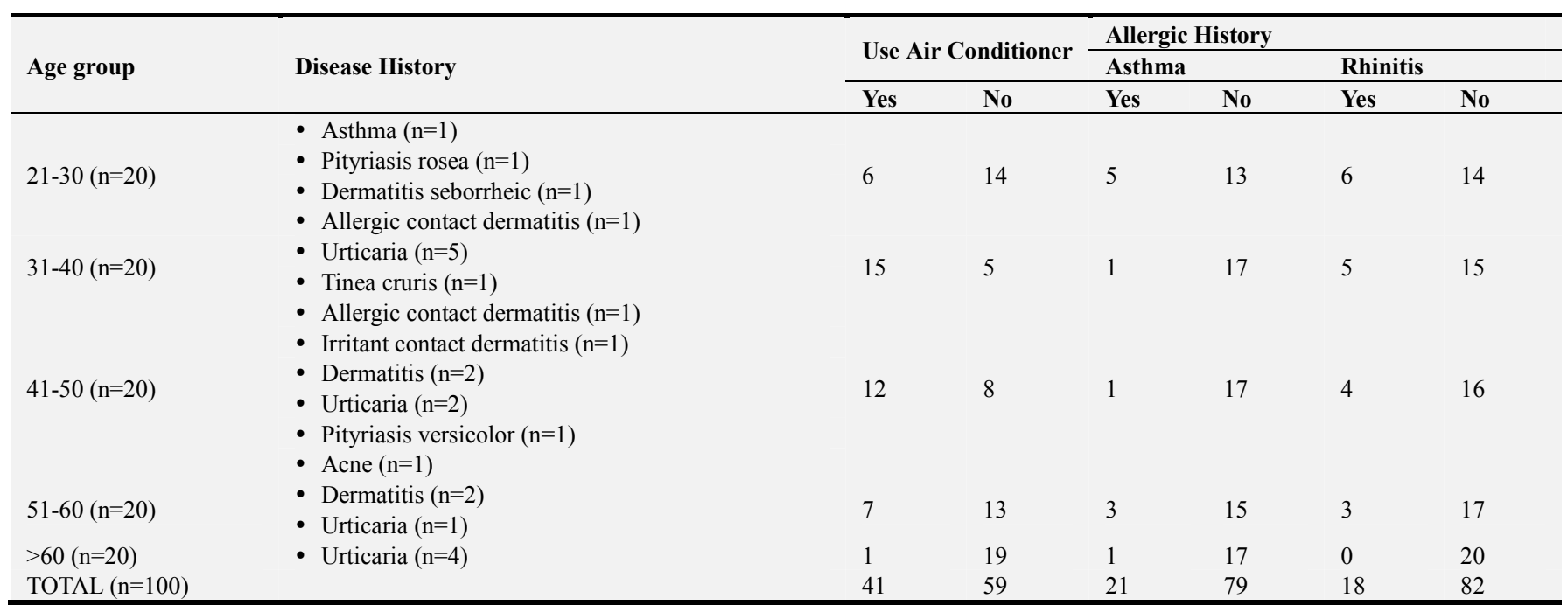

From the table above we can see that there is a correlation between the age groups with nutritional status $(\gamma=-0,235 ; p$ $=0,010)$, in which the older the sample is, the less nutritonal status of the sample would have. There is a correlation between age groups with the sebum levels on the forehead $(\gamma$ $=-0,305 ; \mathrm{p}=0,001)$, where the older the sample is, the less level of sebum on the forehead the sample will have, and also there is a correlation between nutritional status and sebum level on forehead $(\gamma=0,187 ; p=0,032)$, where the better nutritional status, the more sebum on the forehead the sample will have.

\section{Discussion}

Various intrinsic and extrinsic factors, such as age, gender, and regional and ethnic difference affect the skin's physiological and biophysical parameters. Previously, agedependent decreases of transepidermal water loss, stratum corneum (SC) hydration on the forehead, dermal elasticity of the facial skin, and sebum content of nasolabial area have been reported [14].
Many reports on ethnic and regional differences in skin properties have also been published. However, atmospheric condition and climate is an important factor influencing the skin physiology [14].

Dry skin is also known as xerosis, which appears as the moisture content in the stratum corneum is decreased, and if getting worse, the skin becomes weak and vulnerable to infection. The stratum corneum contains $10 \sim 20 \%$ of water from natural moisturizing factors, and if the moisture level becomes below $10 \%$, the corneous tissue becomes thick and the skin texture becomes rough, and the aging accelerates. The extracellular lipids of the stratum corneum function as a water protection film, of which ceramide is over $40 \%$, fatty acids is about $25 \%$, and cholesterol is about $20 \%$. Thus it has been known that if these three compositions change, then the function of protective film can be defected [15].

The human skin is the most active organ among all tissues in the body and maintains the epidermal homeostasis by continuously forming new epidermal cells and repeating differentiation and exfoliation. In particular, the lipid biosynthesis of the epidermis is the critical process of 
forming the skin barrier, and thus the importance of desirable fat intake has been emphasized as a fundamental way of managing dry skin [15].

Skin health status appears to be affected by the interaction of the number of nutrients, not by single nutrient. By considering that the key concept of the quality of life recently is to provide sufficient nutrition from inside and outside the body to maintain skin health and beauty, it should be noted that the functions of nutrients as nutricosmeceuticals are being expanded [15].

Face is covered by lipid film derived from sebum and epidermal lipid. Of the two, sebum, which is secreted by the sebaceous glands, is the major component of the lipid film. Sebum secretions vary individually according to age, sex, inherited traits, and topographical variations of the skin. The amount of facial sebum secretion is an important consideration in facial skin care. Both excessive sebum secretion and reduced sebum secretion are cosmetically undesirable. In general, facial skin types are classified into three types according to an individual's subjective judgment, as: oily, normal, or dry facial skin types [10].

Variations in facial sebum secretion have been known to be related with topographic differences, demographic profiles, inherited factors, and environmental factors [10].

Likewise, with regard to the effects of aging and gender on skin physiology and despite a number of available publications, the results are inconclusive. For example, Marrakchi and Maibach demonstrated that sebum content on the forehead in the aged (aged 66-83 years) is higher than in a younger cohort (aged 24-34 years), while no differences in sebum content between young and old subjects have been observed in another study. In contrast, the sebum content is significantly higher in younger than in older subjects [16], this suggestion is appropriate with the finding in our study.

\section{Conclusion}

From our study we can conclude that there is a correlation between the age groups with nutritional status, correlation between age groups with the sebum levels on the forehead, and also there is a correlation between nutritional status and sebum level on forehead. With a good maintenance of nutritional status in eldery we hope we could restore the dry skin.

\section{References}

[1] Leslie W, Hankey C. Aging, Nutritional Status and Health. Healthcare. 2015; 3: 648-58.

[2] Aghasi M, Motlagh AD, Eshraghian M, Mansouri P. Relationship between Food Security and Receiving Carbohydrate with High Glycemic Index with Acne Infection (Case Study - Control). Switzerland Research Park Journal. 2013; 102: 1986-93.
[3] Boelsma EH, Roza L. Nutritional skin care: health effects of micronutrients and fatty acids. Am J Clin Nutr. 2001:73.

[4] SK LG, Gilchrest B, Paller A, Leffell D, Wolff K. Fitzpatrick's Dermatology in General Medicine. Eighth, editor: McGraw Hill Professional; 2012.

[5] Zouboulis CC, Jourdan E, Picardo M. Acne is an inflammatory disease and alterations of sebum composition initiate acne lesions. JEADV. 2014; 28: 527-32.

[6] Thody AJ, Shuster S. Control and function of sebaceous glands. Physiol Rev. 1989; 69 (2): 383-416.

[7] Cheng J, Russell D. Mammalian wax biosynthesis. II. Expression cloning of wax synthase cDNAs encoding a member of the acyltransferase enzyme family. The Journal of biological chemistry. 2004; 279 (36): 37798-807.

[8] Youn S, Kim S, Hwang I, Park K. Evaluation of facial skin type by sebum secretion: discrepancies between subjective descriptions and sebum secretion. Skin research and technology: official journal of International Society for Bioengineering and the Skin (ISBS) [and] International Society for Digital Imaging of Skin (ISDIS) [and] International Society for Skin Imaging (ISSI). 2002; 8 (3): 168-72.

[9] Pierard G, Marks R, Paye M. EEMCO guidance for the in vivo assessment of skin greasiness. The EEMCO Group 13: Skin Pharmacol Appl Skin Physiol. 2000:372-89.

[10] Youn SW, Na JI, Choi SY, Huh CH, Park KC. Regional and seasonal variations in facial sebum secretions: a proposal for the definition of combination skin type. Skin Research and Technology. 2005; 11: 189-95.

[11] Obesity: Preventing and Managing the Global Epidemic: Report of a WHO Consultation [Internet]. World Health Organization. 2000.

[12] Wang TJ, Russell M, Burastero S, Heymsfield S, Pierson RJ. Asians have lower body mass index (BMI) but higher percent body fat than do whites: comparisons of anthropometric measurements. Am J Clin Nutr. 1994; 60: 23-8.

[13] Hayashi TB, McNeely M, Leonetti D, Kahn S, Fujimoto W. Visceral adiposity, not abdominal subcutaneous fat area, is associated with an increase in future insulin resistance in Japanese Americans. Diabetes. 2008; 57: 1269-75.

[14] Nam GW, Baek JH, Koh JS, Hwang J-K. The seasonal variation in skin hydration, sebum, scaliness, brightness and elasticity in Korean females. Skin Research and Technology. 2014; 0: 1-8.

[15] Bae HS, Choi SI, Ahn HS. Nutritional and antioxidant status by skin types among female adults. 2010; 4 (3): 215-21.

[16] Man MQ, Xin SJ, Song SP, Cho SY, Zhang XJ, Tu CX, et al. Variation of Skin Surface $\mathrm{pH}$, Sebum Content and Stratum Corneum Hydration with Age and Gender in a Large Chinese Population. Skin Pharmacol Physiol. 2009; 22: 190-9. 